\title{
A new way to test the WIMP dark matter models
}

\author{
Wei Cheng, ${ }^{a, b}$ Yuan He, ${ }^{a}$ Jing-Wang Diao, ${ }^{a}$ Yu Pan, ${ }^{a, 1}$ Jun Zeng ${ }^{c, 1}$ \\ and Jia-Wei Zhang ${ }^{d}$ \\ ${ }^{a}$ School of Science, Chongqing University of Posts and Telecommunications, \\ Chongqing 400065, P.R. China \\ ${ }^{b}$ State Key Laboratory of Theoretical Physics, Institute of Theoretical Physics, \\ Chinese Academy of Sciences, Beijing, 100190, P.R. China \\ ${ }^{c}$ Department of Physics, Chongqing University, \\ Chongqing 401331, P.R. China \\ ${ }^{d}$ Department of Physics, Chongqing University of Science and Technology, \\ Chongqing 401331, P.R. China \\ E-mail: chengwei@cqupt.edu.cn, s190601005@stu.cqupt.edu.cn, \\ s200602007@stu.cqupt.en, panyu@cqupt.edu.cn, zengj@cqu.edu.cn, \\ jwzhang@cqust.edu.cn
}

ABSTRACT: In this paper, we investigate the possibility of testing the weakly interacting massive particle (WIMP) dark matter (DM) models by applying the simplest phenomenological model which introduces an interaction term between dark energy (DE) and WIMP DM, i.e., $Q=3 \gamma_{\mathrm{DM}} H \rho_{\mathrm{DM}}$. In general, the coupling strength $\gamma_{\mathrm{DE}}$ is close to 0 as the interaction between DE and WIMP DM is very weak, thus the effect of $\gamma_{\mathrm{DE}}$ on the evolution of $Y$ associated with DM energy density can be safely neglected. Meanwhile, our numerical calculation also indicates that $x_{f} \approx 20$ is associated with DM freeze-out temperature, which is the same as the vanishing interaction scenario. As for DM relic density, it will be magnified by $\frac{2-3 \gamma_{\mathrm{DM}}}{2}\left[2 \pi g_{*} m_{\mathrm{DM}}^{3} /\left(45 s_{0} x_{f}^{3}\right)\right]^{\gamma_{\mathrm{DM}}}$ times, which provides a new way to test WIMP DM models. As an example, we analyze the case in which WIMP DM is a scalar DM. (SGL $+\mathrm{SNe}+\mathrm{Hz})$ and $(\mathrm{CMB}+\mathrm{BAO}+\mathrm{SNe})$ cosmological observations will give $\gamma_{\mathrm{DM}}=0.134_{-0.069}^{+0.17}$ and $\gamma_{\mathrm{DM}}=-0.0008 \pm 0.0016$, respectively. After further considering the constraints from DM direct detection experiment, DM indirect detection experiment, and DM relic density, we find that the allowed parameter space of the scalar DM model will be completely excluded for the former cosmological observations, while it will increase for the latter ones. Those two cosmological observations lead to an almost paradoxical conclusion. Therefore, one could expect more stringent constraints on the WMIP DM models, with the accumulation of more accurate cosmological observations in the near future.

Keywords: Classical Theories of Gravity, Cosmology of Theories beyond the SM

ARXIV EPRINT: 2011.11548

${ }^{1}$ Corresponding author. 


\section{Contents}

1 Introduction 1

2 Dark energy and WIMP dark matter interaction model 2

3 Observational constraints on $\gamma_{\mathrm{DM}} \quad 3$

3.1 Observational data in cosmology 3

3.2 Constraint on $\gamma_{\mathrm{DM}}$

4 WIMP dark matter relic density 9

5 Example - Scalar dark matter as the WIMP dark matter 10

$\begin{array}{lll}5.1 & \text { Scalar dark matter model } & 10\end{array}$

5.2 WIMP DM relic density 10

5.3 DM direct detection 13

5.4 DM indirect detection 14

$\begin{array}{lll}6 & \text { Summary } & 15\end{array}$

\section{Introduction}

Dark energy (DE) and dark matter (DM), which are the main parts of the energy content of the universe, have been firmly established by numerous astronomical and cosmological observations [1-9]. More specifically, the cosmic microwave background (CMB) anisotropies have verified the abundance of DM and DE with remarkable precision at $(26.0 \pm 0.5) \%[10$, 11] and (68.89 \pm 0.56$) \%$ [12] respectively. The usual strategy of particle physicists to deal with the DM is to extend the standard model (SM) of particle physics by adding a new particle that interacts with the SM particles. Based on this interaction, physicists have conducted indirect detection [13-17], direct detection [18-21], and collider detection [22-25] for testing DM models.

There is plentiful literature devoted to the exploration of the interaction between DE and DM to relieve the coincidence problem [26-42]. More specifically, in refs. [43-45], the interaction type between DM and DE is set as $Q=3 H \gamma_{m} \rho_{m}$. After considering the limits from the combination of cosmological data ( $\mathrm{GRBs}+\mathrm{SNe}+\mathrm{BAO}+\mathrm{CMB})$, the coupling strength of DM and DE can be obtained, i.e., $\gamma_{m}=-0.0047 \pm 0.0046$ in $1 \sigma$ errors, which indicates a slight energy transfer from DM to DE. In ref. [46], the interaction between DE and DM (baryonic substances) is set as $3 H \gamma_{c} \rho_{c}\left(3 H \gamma_{b} \rho_{b}\right)$. Taking the bounds from the combination of cosmological data (SNe+BAO+Planck $+\mathrm{Hz}), \gamma_{c}=0.0078 \pm 0.0045$ and $\gamma_{b}=$ $0.0030_{-0.0125}^{+0.0255}$ in $1 \sigma$ errors can be obtained. While using the bounds from the combinations 
of cosmological data $(\mathrm{SNe}+\mathrm{BAO}+\mathrm{WMAP} 9+\mathrm{Hz})$, one can get $\gamma_{c}=0.0068_{-0.0042}^{+0.0043}$ and $\gamma_{b}=$ $0.0025_{-0.0115}^{+0.0235}$ in $1 \sigma$ errors. If one sets $\gamma_{b}=0$, then $\gamma_{c}=0.0095 \pm 0.0031$ in $1 \sigma$ errors within the bounds from the combinations of cosmological data $(\mathrm{SNe}+\mathrm{BAO}+\mathrm{Planck}+\mathrm{Hz})$, and $\gamma_{c}=0.0081 \pm 0.0031$ in $1 \sigma$ errors within the bounds from the combinations of cosmological data $(\mathrm{SNe}+\mathrm{BAO}+\mathrm{WMAP} 9+\mathrm{Hz})$. All predictions of ref. [46] indicate $\gamma_{c}>0$, which means the energy of DE is slightly transferring to that of DM. However, These two references imply that the cosmological data cannot completely exclude the interaction between $\mathrm{DE}$ and DM, and different cosmological data may lead to different prediction about the energy transference between DE and DM.

Motivated by the method of DM Model detection which is based on the interaction between the SM particle and new particles [47-53], we will attempt to develop an alternative DM model verification method based on the new interaction between DM and DE. In the new model, we will restrict ourselves to the Weakly Interacting Massive Particle (WIMP) DM. Specifically, we studied the simplest type of interaction between DE and WIMP DM, namely $Q=3 \gamma_{\mathrm{DM}} H \rho_{\mathrm{M}}$. We deduced the WIMP DM relic density that can be used to examine the WIMP DM models with the help of DM detection experiments.

As an example, we will describe the WIMP DM as a real scalar particle that is the simplest Higgs-portal DM model and has had been comprehensively revisited [54-61]. After considering the WIMP DM relic density, the parameter space of the scalar model will increase with $\gamma_{\mathrm{DM}}<0$, and the smaller $\gamma_{\mathrm{DM}}$, the larger the increased parameter space of the model; while the parameter space will shrink with $\gamma_{\mathrm{DM}}>0$, and the larger $\gamma_{\mathrm{DM}}$, the larger excluded parameter space of this model.

The remaining parts of this paper are organized as follows. In section 2, we construct the DE and WIMP DM models with an interaction term $Q=3 \gamma_{\mathrm{DM}} H \rho_{\mathrm{M}}$. In section 3, we show three observational data in Cosmology and further discuss the coupling strength $\gamma_{\mathrm{DM}}$. In section 4 , the WIMP DM relic density in the new models is calculated. As an example, a scalar DM as the WIMP DM is investigated in detail in section 5. Finally, we will briefly summarize in section 6 .

\section{Dark energy and WIMP dark matter interaction model}

The energy evolution of DE and matter can be described as follows [46, 62]:

$$
\begin{aligned}
\dot{\rho}_{\mathrm{DE}}+3 H\left(\rho_{\mathrm{DE}}+P_{\mathrm{DE}}\right) & =-Q, \\
\dot{\rho}_{\mathrm{M}}+3 H \rho_{\mathrm{M}} & =Q .
\end{aligned}
$$

where $\rho_{\mathrm{DE}}$ and $\rho_{\mathrm{M}}$ are the $\mathrm{DE}$ and matter energy respectively. The interaction term $Q$ describes the interchange of energy with each other, but there is no interaction between them when $Q=0$. Note that those two energies meet the total energy conservation equation: $\dot{\rho}_{\text {tot }}+3 H\left(\rho_{\text {tot }}+P_{\text {tot }}\right)=0$.

We will further divide the matter energy into DM and the usual standard model matter, i.e. $\rho_{\mathrm{M}}=\rho_{\mathrm{DM}}+\rho_{\mathrm{SM}}$. If the DM is the WIMP, then the evolution of $\rho_{\mathrm{DM}}$ can be written as follows:

$$
\dot{\rho}_{\mathrm{DM}}+3 H \rho_{\mathrm{DM}}=Q_{\mathrm{DM}}+\frac{\langle\sigma v\rangle}{m_{\mathrm{DM}}}\left(\rho_{\mathrm{DM}, \mathrm{eq}}^{2}-\rho_{\mathrm{DM}}^{2}\right)
$$


where $\langle\sigma v\rangle$ is the WIMP DM thermal average cross section, and $\rho_{\mathrm{DM}, \text { eq }}$ represents the WIMP DM energy of thermal equilibrium state. Note that eq. (2.2), which is different from other papers, is the most important innovative part of our paper, as both of the WIMP DM annihilation term and the interaction term of DM and DE are considered simultaneously. Usually, cosmologists may not be concerned with the annihilation term $\frac{\langle\sigma v\rangle}{m_{\mathrm{DM}}}\left(\rho_{\mathrm{DM}, \mathrm{eq}}^{2}-\rho_{\mathrm{DM}}^{2}\right)$, while particle physicists do not consider the interaction term $Q_{\mathrm{DM}}$.

Following the common practice in extensive literature, we will consider the usual scenario interaction term $Q=3 \gamma_{\mathrm{DM}} H \rho_{\mathrm{M}}$ and assume $Q_{\mathrm{DM}}=3 \gamma_{\mathrm{DM}} H \rho_{\mathrm{DM}}$. When $\gamma_{\mathrm{DM}}<0$, the energy is transferred from WIMP DM to DE, while for $\gamma_{\mathrm{DM}}>0$, the energy is transferred from DE to WIMP DM, which will affect the feasible parameters of the WIMP DM model. Specifically, the feasible parameters of the WIMP DM model will be shrunken for the case $\gamma_{\mathrm{DM}}>0$, while it will be increased for the case $\gamma_{\mathrm{DM}}<0$. Furthermore, the standard $\Lambda \mathrm{CDM}$ model without interaction between $\mathrm{DE}$ and matter is characterized by $Q=0$, while $Q \neq 0$ represents the non-standard cosmology with interaction between DE and matter.

With the above eq. (2.2) at hand, we can establish a relation between the coupling strength $\gamma_{\text {DM }}$ and the thermal average cross section of WIMP DM through its relic density. As the cosmology observations make a constraint for the coupling strength $\gamma_{\mathrm{DM}}$ through eq. (2.1), we can also establish a relation between the cosmology observations and the thermal average cross section of WIMP DM. We will discuss it in detail in the following parts.

\section{Observational constraints on $\gamma_{\mathrm{DM}}$}

In this section, we will introduce three types of observational data to place constraints on the $\gamma_{\text {DM }}$ interaction dark energy model parameters, i.e., 130 updated galaxy-scale strong gravitational lensing sample (SGL) [63, 64] with redshift from 0.197 to 2.834, Hubble parameter data $(\mathrm{Hz})$ [65], and Pantheon 1048 Ia supernovae sample (SNe) discovered by the Pan-STARRSI Medium Deep Survey [66].

\subsection{Observational data in cosmology}

In the electromagnetic and gravitational wave domain [63, 64, 67, 68], strong gravitational lensing has been widely used in precision cosmology, concerning accurate determination of cosmological parameters [69-73] and cosmic opacity at higher redshifts [74], constraints on the velocity dispersion function [75-77] and dark matter distribution in early-type galaxies [78], direst tests of Parametrized Post-Newtonian gravity [79] and the validity of the FLRW metric [80, 81], as well as precise measurements of the speed of light [82-84] with galaxy-scale strong lensing systems.

Considering the influence of some unknown system errors on the SGL data, Chen et al. compiled 161 galaxy-scale strong gravitational lensing sample system which include the gravitational lensing and stellar velocity dispersion measurements. They selected those samples from early-type galaxies with $E / S 0$ morphologies with strict criteria to satisfy the assumption of spherical symmetry on the lens mass model. In their model, they discuss the slope of the total mass density profile $\gamma$ considering three parameterizations $\left(\gamma=\gamma_{0}\right.$, 
$\gamma=\gamma_{0}+\gamma_{z} \times z_{l}$ and $\gamma=\gamma_{0}+\gamma_{z} \times z_{l}+\gamma_{s} \times \log \tilde{\sum}$.), the luminosity density slope $\delta$ with an observable parameter for each lens and the orbit anisotropy parameter $\beta$ treated as a nuisance parameter and marginalized over with a Gaussian prior $\beta=0.18 \pm 0.13$ [64]. Moreover, because of the high-resolution HST imaging data needed, they finally chose 130 galaxy-scale strong gravitational lensing data from 161 samples, which are separated from the Sloan Lens ACS(SLACS) and others surveys. The specific selection methods can be found in the literature [64].

When considering the effect of aperture size on the velocity dispersion of the lens galaxy, a more appropriate choice for the radius is $R_{\text {eff }} / 2$ with $R_{\text {eff }}$ being the half-light radius of the lens galaxy, because $R_{\text {eff }}$ matches the Einstein radius well [85]. Choose $R_{\text {eff }} / 2$ as the radius to get the velocity dispersion $\sigma_{e 2}$, according to the regulations, the observational expression of velocity dispersion is as follows [63]

$$
\sigma_{\mathrm{obs}} \equiv \sigma_{e 2}=\sigma_{a p}\left[\theta_{\mathrm{eff}} /\left(2 \theta_{a p}\right)\right]^{\eta}
$$

where $\sigma_{a p}$ is velocity dispersion, $\theta_{\text {eff }}=R_{\text {eff }} / D_{l}$, the correction factor $\eta=-0.066 \pm$ 0.035 [86], and $\theta_{\text {ap }} \approx 1.025 \times \sqrt{\left(\theta_{x} \theta_{y} / \pi\right)}$ with $\theta_{x}$ and $\theta_{y}$ being the angular sizes of width and length of the rectangular aperture respectively [87].

The total error of the actual velocity dispersion is

$$
\left(\Delta \sigma_{\text {tot }}\right)^{2}=\left(\Delta \sigma_{\text {stat }}\right)^{2}+\left(\Delta \sigma_{\text {sys }}\right)^{2}+\left(\Delta \sigma_{\mathrm{AC}}\right)^{2},
$$

where $\Delta \sigma_{\text {stal }}$, propagated from the measurement error of $\sigma_{\text {ap }}$, is the statistical error, $\Delta \sigma_{\text {gys }}$ is systematic error, and the error $\Delta \sigma_{\mathrm{AC}}$ is propagated from the uncertainty of $\eta$ due to the aperture correction.

The theoretical expression of the velocity dispersion can be written as [78]

$$
\sigma_{\mathrm{th}}=\sqrt{\frac{c^{2}}{2 \sqrt{\pi}} \frac{D_{s}}{D_{l s}} \theta_{E} \frac{3-\delta}{(\xi-2 \beta)(3-\xi)} F(\gamma, \delta, \beta)\left(\frac{\theta_{\mathrm{eff}}}{2 \theta_{\mathrm{E}}}\right)^{(2-\gamma)}}
$$

where $D_{l s}$ is the angular diameter distance between lens and source, $D_{s}$ is that between observer and source, which are dependent on the cosmological model. The cosmological model enters into the theoretical observable not through a distance measure directly, but rather through a distance ratio: $\frac{D_{s}\left(z_{s} ; \mathbf{p}, H_{0}\right)}{D_{l s}\left(z_{l}, z_{s} ; \mathbf{p}, H_{0}\right)}$. The expressions for both $D_{s}\left(z_{s} ; \mathbf{p}, H_{0}\right)$ and $D_{l s}\left(z_{l}, z_{s} ; \mathbf{p}, H_{0}\right)$ are as follows:

$$
\begin{aligned}
D_{s}\left(z_{s} ; \mathbf{p}, H_{0}\right) & =\frac{c}{H_{0}\left(1+z_{s}\right)} \int_{0}^{z_{s}} \frac{d z}{E(z ; \mathbf{p})}, \\
D_{l s}\left(z_{l}, z_{s} ; \mathbf{p}, H_{0}\right) & =\frac{c}{H_{0}\left(1+z_{s}\right)} \int_{z_{l}}^{z_{s}} \frac{d z}{E(z ; \mathbf{p})},
\end{aligned}
$$

where $E(z ; \mathbf{p})$ is the cosmological background of the $\gamma_{\text {DM }}$ model that has the following form

$$
E^{2}(z ; \mathbf{p})=\frac{w_{x} \Omega_{m}}{\gamma_{\mathrm{DM}}+w_{x}}(1+z)^{3\left(1-\gamma_{\mathrm{DM}}\right)}+\left(1-\frac{w_{x} \Omega_{m}}{\gamma_{\mathrm{DM}}+w_{x}}\right)(1+z)^{3\left(1+w_{x}\right)}
$$

where $\mathbf{p}$ is the model parameters, i.e., $\mathbf{p}=\left(\Omega_{m}, \omega_{x}, \gamma_{\mathrm{DM}}\right)$. 
The function $F(\gamma, \delta, \beta)$ in eq. (3.3) can be expressed as [63]

$$
F(\gamma, \delta, \beta)=\left[\frac{\Gamma[(\xi-1) / 2]}{\Gamma(\xi / 2)}-\beta \frac{\Gamma[(\xi+1) / 2]}{\Gamma[(\xi+2) / 2]}\right] \frac{\Gamma(\gamma / 2) \Gamma(\delta / 2)}{\Gamma[(\gamma-1) / 2] \Gamma[(\delta-1) / 2]}
$$

where $\xi=\gamma+\delta-2$. In this paper, we consider the dependence of $\gamma$ on lens redshift $z_{l}$ and the dependence of redshift on surface mass density (i.e., P3 in the literature [64]). The expression of $\gamma$ is as follows:

$$
\gamma=\gamma_{0}+\gamma_{z} \times z_{l}+\gamma_{s} \times \log \tilde{\Sigma}
$$

where $\tilde{\Sigma}$ is the surface mass density,

$$
\tilde{\Sigma}=\frac{\left(\sigma_{\mathrm{obs}} / 100 \mathrm{kms}^{-1}\right)^{2}}{R_{\mathrm{eff}} / 10 h^{-1} \mathrm{kpc}}
$$

$h$ is from the Hubble constant $H_{0}=100 h \mathrm{~km}^{-1} \mathrm{Mpc}^{-1}$.

In addition, we also consider the latest Pantheon supernova sample [66]

$$
\mu=M+5 \log \frac{d_{L}}{\mathrm{pc}}+25
$$

where

$$
d_{L}=\frac{(1+z)}{H_{0}} \int_{0}^{z} \frac{d z^{\prime}}{E\left(z^{\prime}\right)}
$$

Finally, the 31 Hubble parameter $(\mathrm{Hz})$ samples from the differential age method are also used [65], focusing on model-independent reconstruction of cosmological distances [88, 89] and the spatial curvature of the Universe [90, 91], as well as statistical analysis of parameterized $\operatorname{Om}(z)$ diagnostics in light of recent observations [92, 93].

\subsection{Constraint on $\gamma_{\mathrm{DM}}$}

The parameters of the $\gamma_{\mathrm{DM}}$ model are constrained by using (SGL+SNe+Hz) sample combination through Markov chain Monte Carlo (MCMC) method [94], and fitted by the minimum likelihood method of $\chi^{2}$. The final $\chi_{\text {All }}^{2}$ is given by the following function:

$$
\chi_{\mathrm{All}}^{2}=\chi_{\mathrm{SGL}}^{2}+\chi_{\mathrm{SNe}}^{2}+\chi_{\mathrm{Hz}}^{2} .
$$

where $\chi_{\mathrm{SGL}}^{2}, \chi_{\mathrm{SNe}}^{2}$, and $\chi_{\mathrm{Hz}}^{2}$ can be expressed as follows:

$$
\begin{aligned}
\chi_{\mathrm{SGL}}^{2} & =\sum_{i=1}^{130}\left(\frac{\sigma_{\mathrm{th}}-\sigma_{\mathrm{obs}}}{\Delta \sigma_{\mathrm{tot}}}\right)^{2}, \\
\chi_{\mathrm{SNe}}^{2} & =\sum_{i=1}^{1048}\left(\frac{\mu_{t h}-\mu_{\mathrm{obs}}}{\sigma_{\mu}}\right)^{2}, \\
\chi_{\mathrm{Hz}}^{2} & =\sum_{i=1}^{31}\left(\frac{H z_{t h}-H z_{\mathrm{obs}}}{\sigma_{\mathrm{Hz}}}\right)^{2} .
\end{aligned}
$$




\begin{tabular}{|ccc|}
\hline Parameters & $1 \sigma$ & $2 \sigma$ \\
\hline$\Omega_{m}$ & $0.445_{-0.076}^{+0.15}$ & $0.445_{-0.23}^{+0.19}$ \\
$\omega_{x}$ & $-1.59_{-0.35}^{+0.54}$ & $-1.59_{-0.82}^{+0.71}$ \\
$\gamma_{\mathrm{DM}}$ & $0.134_{-0.069}^{+0.17}$ & $0.134_{-0.32}^{+0.23}$ \\
$H_{0}$ & $71.54 \pm 0.39$ & $71.54_{-0.74}^{+0.78}$ \\
$\gamma_{0}$ & $1.237 \pm 0.067$ & $1.237_{-0.15}^{+0.15}$ \\
$\gamma_{z}$ & $-0.218 \pm 0.074$ & $-0.218_{-0.15}^{+0.14}$ \\
$\gamma_{s}$ & $0.652 \pm 0.055$ & $0.652_{-0.11}^{+0.11}$ \\
\hline
\end{tabular}

Table 1. The best values $\left(\Omega_{m}, \omega_{x}, \gamma_{\mathrm{DM}}, H_{0}, \gamma_{0}, \gamma_{z}, \gamma_{s}\right)$ and their $2 \sigma$ errors of the $\gamma_{\mathrm{DM}}$ model parameters are obtained by using $\mathrm{SGL}+\mathrm{SNe}+\mathrm{Hz}$ observational data combination.

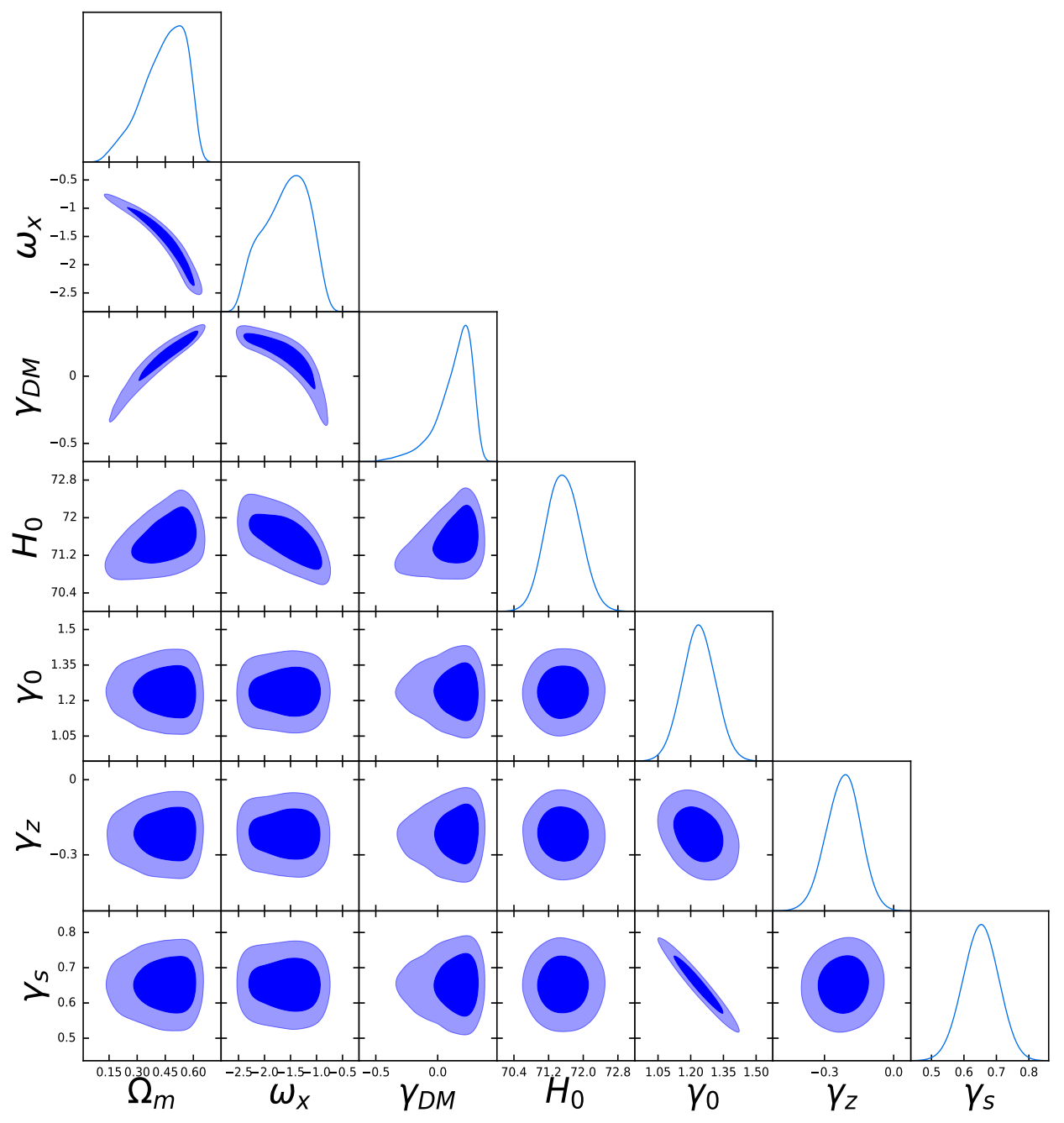

Figure 1. The $2 \mathrm{D}$ contour line are obtained by using $\mathrm{SGL}+\mathrm{SNe}+\mathrm{Hz}$. 
The best values of each parameter $\left(\Omega_{m}, \omega_{x}, \gamma_{\mathrm{DM}}, H_{0}, \gamma_{0}, \gamma_{z}, \gamma_{s}\right)$ and their $2 \sigma$ error results are shown in table. 1 . The $2 \mathrm{D}$ contour line is shown in figure 1.

We can see that the interaction factor $\gamma_{\mathrm{DM}}\left(\gamma_{\mathrm{DM}}=0.134_{-0.069}^{+0.17}\right)$ is not zero within the $1 \sigma$ error range and the best value is also $\gamma_{\mathrm{DM}}>0$ from table 1 , which indicate there is a trend of conversion from DE to DM. The coincidence problem may be alleviated slightly at the range of $1 \sigma$ error. In addition, the best value of $H_{0}$ is $H_{0}=71.54 \mathrm{kms}^{-1} \mathrm{Mpc}^{-1}$, which is larger than $H_{0}=67.4 \mathrm{kms}^{-1} \mathrm{Mpc}^{-1}$ given by Plank2018 [12] and smaller than $H_{0}=74.03 \mathrm{kms}^{-1} \mathrm{Mpc}^{-1}$ given by Pantheon [95], which alleviates the conflict problem of $H_{0}$ to some extent. It shows that the $\gamma_{\mathrm{DM}}$ model has the ability to alleviate the tension problem of Hubble constant $H_{0}$ which is consistent with the results in reference [96]. We also note that the coupling parameter $\gamma_{\mathrm{DM}}$ is correlated with all other model parameters $\Omega_{m}, \omega_{X}$ and $H_{0}$, and the cosmological constant model $(\Lambda \mathrm{CDM})\left(\gamma_{\mathrm{DM}}=0, \omega_{X}=0\right)$ is contained in the $2 \sigma$ confidence region. The matter density parameter $\Omega_{m}$ is also consistent with the result from other observational data in the $2 \sigma$ confidence region $[12,95]$ although the best value is slightly larger.

For the large $\gamma_{\mathrm{DM}}$ obtained from the (SGL $+\mathrm{SNe}+\mathrm{Hz}$ ) data analysis, we analyzed the SGL data separately, which leads to $\gamma_{\mathrm{DM}}=0.15_{-0.10}^{+0.35}(1 \sigma)_{-0.51}^{+0.36}(2 \sigma)$. Compared with $\gamma_{\mathrm{DM}}=$ $0.134_{-0.069}^{+0.17}(1 \sigma)_{-0.32}^{+0.23}(2 \sigma)$ obtained by $(\mathrm{SGL}+\mathrm{SNe}+\mathrm{Hz})$ data analysis, one can conclude that SGL is the main reason for the large $\gamma_{\mathrm{DM}}$.

For comparison with the standard "BAO+SNe+CMB", we also consider other combinations of these data for comparison $(\mathrm{CMB}+\mathrm{BAO}, \mathrm{CMB}+\mathrm{BAO}+\mathrm{SNe}$, $\mathrm{CMB}+\mathrm{BAO}+\mathrm{SNe}+\mathrm{GRB}, \mathrm{CMB}+\mathrm{BAO}+\mathrm{SNe}+\mathrm{GRB}+\mathrm{SGL})$. The total results are shown in table 2 and in figure 2. In which, the CMB measurement from Planck TT, TE, EE+lowE data are released in 2018 [97], and the BAO data set used in our analysis includes five lowerredshift BAO measurements from galaxy surveys at the redshifts $z=0.106 \sim 0.61$ regions and one higher-redshift BAO measurements from $\operatorname{Ly} \alpha$ forest $(\operatorname{Ly} \alpha)$ data at $z=2.35$. For the lower-redshift BAO observations, we turn to the latest measurements of the acoustic-scale distance ratio from 6dFGS at $z=0.106$ [98], the SDSS Data Release 7 Main Galaxy sample at $z=0.15$ [99], three data obtained by the BOSS DR12 galaxies $(z=0.38,0.51,0.61)[100]$, and the higher-redshift BAO measurement is quasar-ly $\alpha$ cross in combination with ly $\alpha$ auto combination at $z=2.35$ [101].

Firstly, from the two sets of standard data $(\mathrm{CMB}+\mathrm{BAO})$ and $(\mathrm{CMB}+\mathrm{BAO}+\mathrm{SNe})$ in table 2 , one can find that the model parameters $\left(\Omega_{m}, w_{\mathrm{X}}, \gamma_{\mathrm{DM}}, H_{0}\right)$ are confined tightly. Secondly, according to the table. 2, the GRB data had little effect on the results, which is consistent with our previous ref. [44]. Specifically, the best value of $\gamma_{\mathrm{DM}}, \Omega_{m}$ and $H_{0}$ change slightly and the $\gamma_{\mathrm{DM}}$ results constrained by the $(\mathrm{CMB}+\mathrm{BAO}+\mathrm{SNe})$ and $(\mathrm{CMB}+\mathrm{BAO}+\mathrm{SNe}+\mathrm{GRB})$ are almost the same at $1 \sigma$ error region. Thirdly, we combine the SGL data with $(\mathrm{CMB}+\mathrm{BAO}+\mathrm{SNe}+\mathrm{GRB})$ data to confine the value of $\gamma_{\mathrm{DM}}$, and the results are also listed in table 2 and figure 2. Although the analysis of SGL data alone leads $\gamma_{\mathrm{DM}}$ to be larger, the value of $\gamma_{\mathrm{DM}}$ from the analysis of $(\mathrm{CMB}+\mathrm{BAO})$ or $(\mathrm{CMB}+\mathrm{BAO}+\mathrm{SNe})$ is close to that of the combined analysis of SGL and (GRBs+SNe+BAO+CMB), which indicates that the data $(\mathrm{CMB}+\mathrm{BAO})$ is the strongest and standard confine. In addition, the best value $\gamma_{\mathrm{DM}}$ and the $1 \sigma$ error region from the analysis of SGL data are excluded by the stan- 


\begin{tabular}{|c|cccc|}
\hline Parameters & CMB+BAO & $\mathrm{CMB}+\mathrm{BAO}+\mathrm{SNe}$ & $\mathrm{CMB}+\mathrm{BAO}+\mathrm{SNe}+\mathrm{GRB}$ & $\mathrm{CMB}+\mathrm{BAO}+\mathrm{SNe}+\mathrm{GRB}+\mathrm{SGL}$ \\
\hline$\Omega_{m}$ & $0.310 \pm 0.012$ & $0.3065 \pm 0.0086$ & $0.3065 \pm 0.0086$ & $0.2952 \pm 0.0084$ \\
$w_{\mathrm{X}}$ & $-0.993_{-0.049}^{+0.056}$ & $-1.008 \pm 0.035$ & $-1.007 \pm 0.035$ & $-1.058 \pm 0.035$ \\
$\gamma_{\mathrm{DM}}$ & $-0.0007 \pm 0.0016$ & $-0.0008 \pm 0.0016$ & $-0.0008 \pm 0.0016$ & $-0.0009 \pm 0.0016$ \\
$H_{0}$ & $68.0 \pm 1.5$ & $68.4 \pm 1.2$ & $68.4 \pm 1.2$ & $69.8 \pm 1.2$ \\
\hline$\gamma_{0}$ & - & - & - & $2.75 \pm 0.13$ \\
$\gamma_{z}$ & - & - & - & $-0.412_{-0.092}^{+0.10}$ \\
$\gamma_{s}$ & - & - & - & $0.256 \pm 0.054$ \\
\hline
\end{tabular}

Table 2. The best values and their $1 \sigma$ errors of the $\gamma_{\mathrm{DM}}$ model parameters are obtained by using $(\mathrm{CMB}+\mathrm{BAO}),(\mathrm{CMB}+\mathrm{BAO}+\mathrm{SNe}), \quad(\mathrm{CMB}+\mathrm{BAO}+\mathrm{SNe}+\mathrm{GRB})$ and $(\mathrm{CMB}+\mathrm{BAO}+\mathrm{SNe}+\mathrm{GRB}+\mathrm{SGL})$ observation combinations.

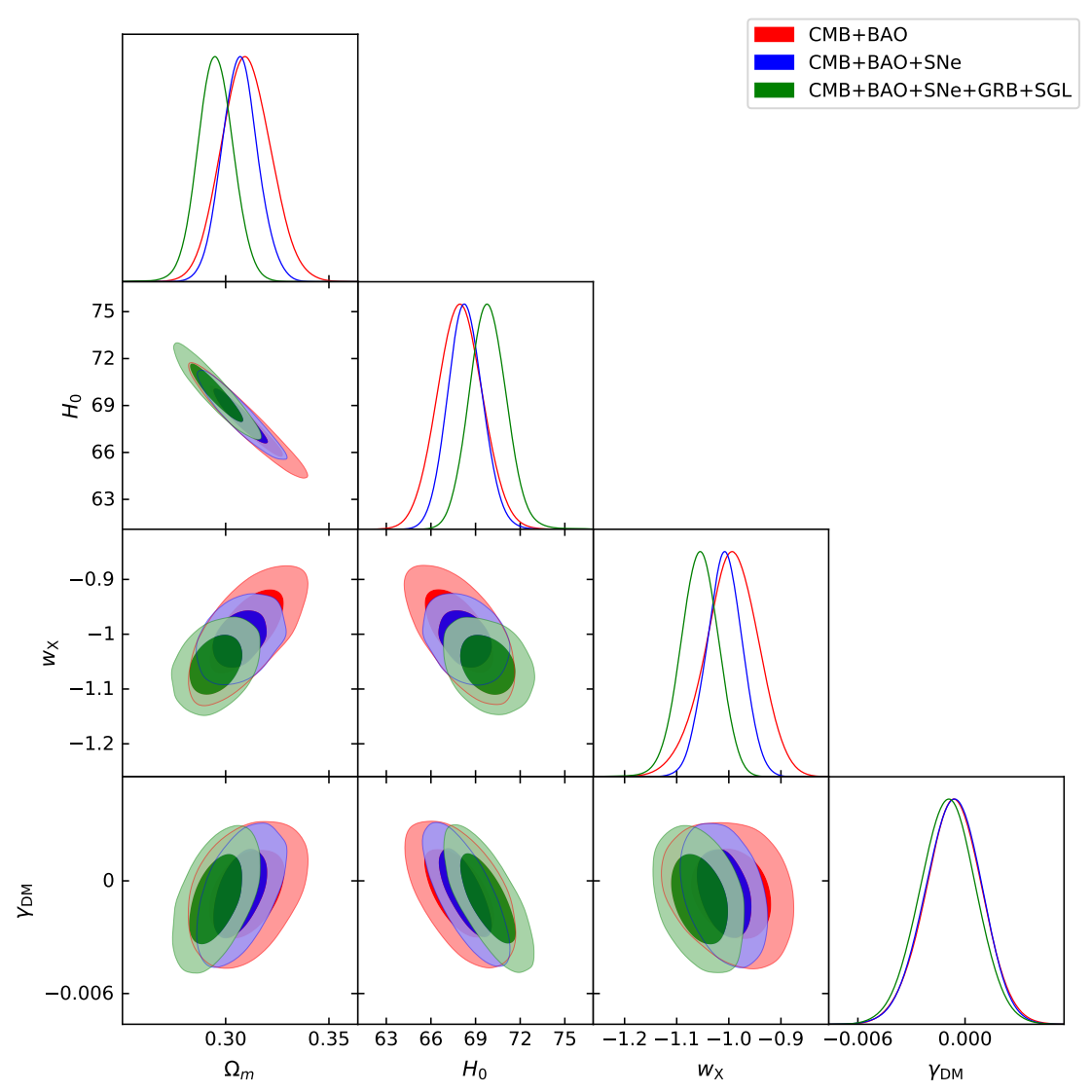

Figure 2. The $2 \mathrm{D}$ contour line are obtained by using $(\mathrm{CMB}+\mathrm{BAO}),(\mathrm{CMB}+\mathrm{BAO}+\mathrm{SNe})$ and $(\mathrm{CMB}+\mathrm{BAO}+\mathrm{SNe}+\mathrm{GRB}+\mathrm{SGL})$.

dard "BAO $+\mathrm{SNe}+\mathrm{CMB}$ ", but the results are consistent with each other within $1 \sigma$ region when they are constrained by combined data (CMB+BAO+SNe+GRB+SGL). The tension of the best value $\gamma_{\mathrm{DM}}$ obtained from SGL data and the standard "BAO+SNe+CMB" may be attributed to SGL data with lower redshift compared with CMB data with high redshift. Although the best values of $\gamma_{\mathrm{DM}}$ from SGL are excluded by the standard "BAO $+\mathrm{SNe}+\mathrm{CMB}$ ", $\gamma_{\mathrm{DM}}=0$ is within the $2 \sigma$ confidence region, which is consistent with the conclusion of the standard "BAO+SNe+CMB" analysis. 


\section{WIMP dark matter relic density}

The evolution of DM can be described by:

$$
\dot{\rho}_{\mathrm{DM}}+3 H \rho_{\mathrm{DM}}=3 \gamma_{\mathrm{DM}} H \rho_{\mathrm{DM}}+\frac{\langle\sigma v\rangle}{m_{\mathrm{DM}}}\left(\rho_{\mathrm{DM}, \mathrm{eq}}^{2}-\rho_{\mathrm{DM}}^{2}\right),
$$

where $\rho_{\mathrm{DM}}=m_{\mathrm{DM}} n_{\mathrm{DM}}$ with $n_{\mathrm{DM}}$ being the DM number density. The above evolution of DM equation contains the core information of DM, such as the freeze-out parameter $x_{f}=m_{\mathrm{DM}} / T$ with $T$ being the DM freeze-out temperature and the DM relic density. In the following, we will go through the derivation of these quantities in detail in this new model [102].

By making a substitution $x=m_{\mathrm{DM}} / T$ and $Y=n_{\mathrm{DM}} S^{\gamma_{\mathrm{DM}}-1}$ with $S$ being the entropy of the universe, the eq. (4.1) with the help of relation $d S(T) / d t+3 S(T) H=0$ and $\langle\sigma v\rangle=$ $\langle\sigma v\rangle_{0} x^{-n}$ can be achieved:

$$
\frac{d Y}{d x}=\frac{\langle\sigma v\rangle_{0} x^{3 \gamma_{\mathrm{DM}}-n-2}}{H(m) S(m)^{\gamma_{\mathrm{DM}}-1}}\left(Y_{\mathrm{eq}}^{2}-Y^{2}\right)
$$

where $H(m)=H x^{2}$ and $S(m)=S(T) x^{3}$.

For the further study, we set $y=\frac{\langle\sigma v\rangle_{0} n_{\mathrm{DM}} S^{\gamma_{\mathrm{DM}}-1}}{H(m) S(m)^{\gamma} \mathrm{DM}^{-1}}$, thus $y_{\mathrm{eq}}=\frac{\langle\sigma v\rangle_{0} n_{\mathrm{DM}, \mathrm{eq}} S^{\gamma_{\mathrm{DM}}}-1}{H(m) S(m)^{\gamma} \mathrm{DM}^{-1}}$ with $n_{\mathrm{DM}, \mathrm{eq}}=g\left(\frac{m T}{2 \pi}\right)^{3 / 2} \exp (-m / T)$. We can further transform eq. (4.1) as:

$$
\frac{d y}{d x}=\frac{1}{x^{3-3 \gamma_{\mathrm{DM}}}}\left(y_{\mathrm{eq}}^{2}-y^{2}\right) .
$$

The interaction strength $\gamma_{\mathrm{DM}}$ between DE and DM, though generally small, will be set to $\gamma_{\mathrm{DM}}<|0.05|$ to test the $x_{f}$. Taking the usual inputs, ${ }^{1}$ we plot $Y$ as a function of $x$ in figure 3 . We find that $x_{f}$ hardly changes in $\gamma_{\mathrm{DM}}<|0.05|$, i.e., $x_{f} \approx 20$.

As the WIMP DM relic density is given by:

$$
\Omega=\frac{\rho_{D M_{0}}}{\rho_{\text {crit }}}
$$

where the critical energy density $\rho_{\text {crit }}=\frac{3 H_{0}}{8 \pi G_{N}}$ and the energy density of DM today can be expressed as follows:

$$
\rho_{D M_{0}}=m_{\mathrm{DM}} Y_{\infty} s_{0}^{1-\gamma_{\mathrm{DM}}}
$$

where $Y_{\infty}=\frac{H(m) S(m)^{\gamma_{\mathrm{DM}}-1}}{\langle\sigma v\rangle_{0}}\left(n+1-3 \gamma_{\mathrm{DM}}\right) x_{f}^{n+1-3 \gamma_{\mathrm{DM}}}$, which can be obtained by solving eq. (4.2), and the entropy of the universe today is $S_{0}=2890 \mathrm{~cm}^{-3}$.

Finally, the WIMP DM relic density can be obtained with $n=1$ as follows:

$$
\Omega h^{2}=0.169 \times x_{f} \sqrt{\frac{100}{g_{*}}} \frac{10^{-10} G e V^{-2}}{\langle\sigma v\rangle} \frac{2-3 \gamma_{\mathrm{DM}}}{2}\left(\frac{2 \pi g_{*} m_{\mathrm{DM}}^{3}}{45 S_{0} x_{f}^{3}}\right)^{\gamma_{\mathrm{DM}}} .
$$

\footnotetext{
${ }^{1} \mathrm{DM}$ mass is $m_{\mathrm{DM}}=1000 \mathrm{GeV}$, reduced Planck mass is $M_{P l}=(8 \pi G)^{-1 / 2}=2.44 \times 10^{18} \mathrm{GeV}$, the number of degrees of freedom for the field is $g_{*}=100$, and the cross section is $\langle\sigma v\rangle_{0}=10^{-10} \mathrm{GeV}^{-2}$.
} 


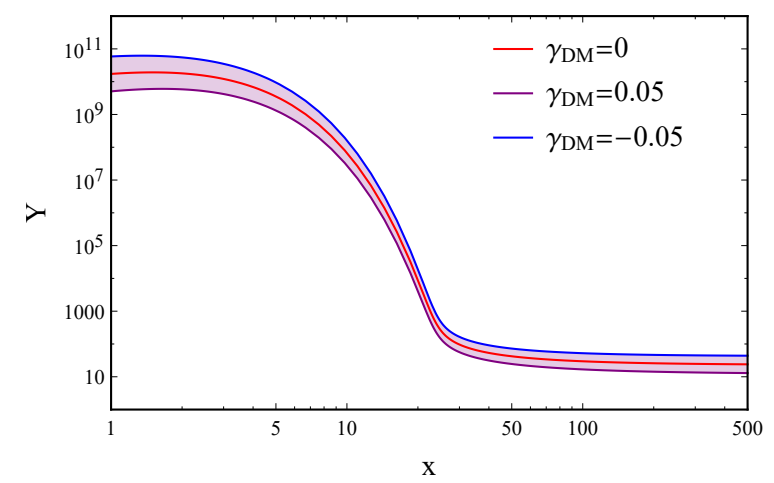

Figure 3. The plot of $Y$ by $x$ for several values of $\gamma_{\mathrm{DM}}$.

\section{Example - Scalar dark matter as the WIMP dark matter}

\subsection{Scalar dark matter model}

Let us firstly discuss the Higgs potential with a real scalar DM, which can be written as [60]:

$$
V(\Phi, \mathcal{S})=-\mu_{h}^{2}|\Phi|^{2}+\lambda_{h}|\Phi|^{4}+\frac{1}{2} \mu_{\mathcal{S}}^{2} \mathcal{S}^{2}+\frac{1}{2} \lambda_{\mathcal{S}} \mathcal{S}^{4}+\frac{1}{2} \lambda_{h \mathcal{S}}|\Phi|^{2} \mathcal{S}^{2},
$$

where $\Phi$ and $\mathcal{S}$ are the SM Higgs and the real scalar DM, respectively. The interaction between DM sector and SM Higgs is described by the last term, and the coupling constant $\lambda_{h \mathcal{S}}$ reflect the strength of this interaction. The cubic term of $\mathcal{S}$ is eliminated due to the even $Z_{2}$ parity symmetry of the real scalar DM.

After the electroweak symmetry spontaneously breaks, the dark sector of eq. (5.1) can be written as,

$$
V(h, \mathcal{S})=\frac{m_{\mathcal{S}}^{2}}{2} \mathcal{S}^{2}+\frac{\lambda_{\mathcal{S}}}{2} \mathcal{S}^{4}+\frac{\lambda_{h \mathcal{S}} v}{2} \mathcal{S}^{2} h+\frac{\lambda_{h \mathcal{S}}}{4} \mathcal{S}^{2} h^{2},
$$

where the square of DM mass is $m_{\mathcal{S}}^{2}=\mu_{\mathcal{S}}^{2}+\lambda_{h \mathcal{S}} v^{2} / 2$ with the electroweak scale $v \simeq 246 \mathrm{GeV}$.

\subsection{WIMP DM relic density}

In this part, we will show the calculation technique in detail for the scalar DM thermal average annihilation cross section that is the important component of the scalar DM relic density, which can be written as [103]:

$$
\langle\sigma v\rangle=\frac{x}{8 m_{\mathcal{S}}^{5} K_{2}^{2}(x)} \int_{4 m_{\mathcal{S}}^{2}}^{\infty}\left(s-4 m_{\mathcal{S}}^{2}\right) \sqrt{s} K_{1}\left(x \sqrt{s} / m_{\mathcal{S}}\right) \sigma \mathrm{d} s .
$$

Here $x=m_{\mathcal{S}} / T$ ( $T$ is the temperature), $K_{1}(y)$ and $K_{2}(y)$ are the modified Bessel function of the second kind, and $s$ is the square of the center-of-mass energy. According to our scalar DM model eq. (5.1), there are four kinds of annihilation feynman diagrams which are shown in figure 4, then scalar dark matter annihilation cross section $\sigma$ can be obtained as,

$$
\sigma=\sigma^{f \bar{f}}+\sigma^{W^{+} W^{-}}+\sigma^{Z Z}+\sigma^{h h} .
$$




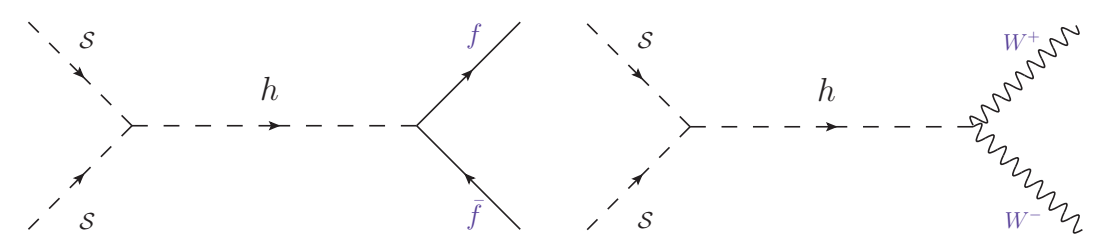

(a)

(b)

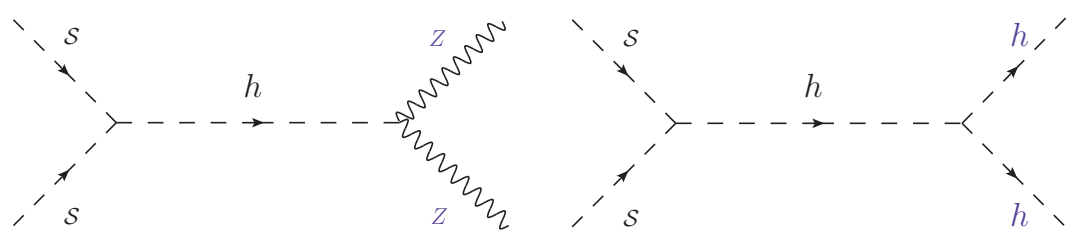

(c)

$(d)$

Figure 4. Feynman diagrams for the scalar dark matter annihilation.

The cross section $\sigma^{X X}$ can be cast into the following form:

$$
\sigma^{X X}=\lambda_{h \mathcal{S}}^{2} \nu^{2}\left|D_{h}(s)\right|^{2} \Gamma_{h \rightarrow X X}(s),
$$

where,

$$
\left|D_{h}(s)\right|^{2} \equiv \frac{1}{\left(s-m_{h}^{2}\right)^{2}+m_{h}^{2} \Gamma_{h}^{2}} .
$$

After utilizing the standard quantum field theory calculations, the cross section in right hands of eq. (5.4) can be obtained. More explicitly, we give the final result as follows:

$$
\begin{aligned}
\sigma^{f \bar{f}} & =N_{C}^{f} \sum_{f} \frac{\lambda_{h \mathcal{S}}^{2} m_{f}^{2}\left(1-4 m_{f}^{2} / s\right)^{3 / 2}}{8 \pi\left(m_{h}^{2} \Gamma_{h}^{2}+\left(s-m_{h}^{2}\right)^{2}\right)}, \\
\sigma^{W^{+} W^{-}} & =\frac{\lambda_{h \mathcal{S}}^{2}\left(s+12 \frac{m_{W}^{4}}{s}-4 m_{W}^{2}\right) \sqrt{1-4 m_{W}^{2} / s}}{16 \pi\left(m_{h}^{2} \Gamma_{h}^{2}+\left(s-m_{h}^{2}\right)^{2}\right)}, \\
\sigma^{Z Z} & =\frac{\lambda_{h \mathcal{S}}^{2}\left(s+12 \frac{m_{Z}^{4}}{s}-4 m_{Z}^{2}\right) \sqrt{1-4 m_{Z}^{2} / s}}{32 \pi\left(m_{h}^{2} \Gamma_{h}^{2}+\left(s-m_{h}^{2}\right)^{2}\right)} \\
\sigma^{h h} & =\frac{9 \lambda_{h \mathcal{S}}^{2} m_{h}^{4} \sqrt{1-4 m_{h}^{2} / s}}{32 \pi s\left(m_{h}^{2} \Gamma_{h}^{2}+\left(s-m_{h}^{2}\right)^{2}\right.} .
\end{aligned}
$$

These analytic annihilation formulas indicate that $s$ must be more than twice the mass of a particle before the corresponding annihilation channel can be opened. For example, if $2 m_{W}>s>2 m_{f \bar{f}}$, then only $\mathcal{S} \mathcal{S} \rightarrow f \bar{f}$ annihilation will be opened.

The newest Plank and WMAP data have measured the DM relic density in high precision [12]:

$$
\Omega h^{2}=0.1198 \pm 0.0012
$$




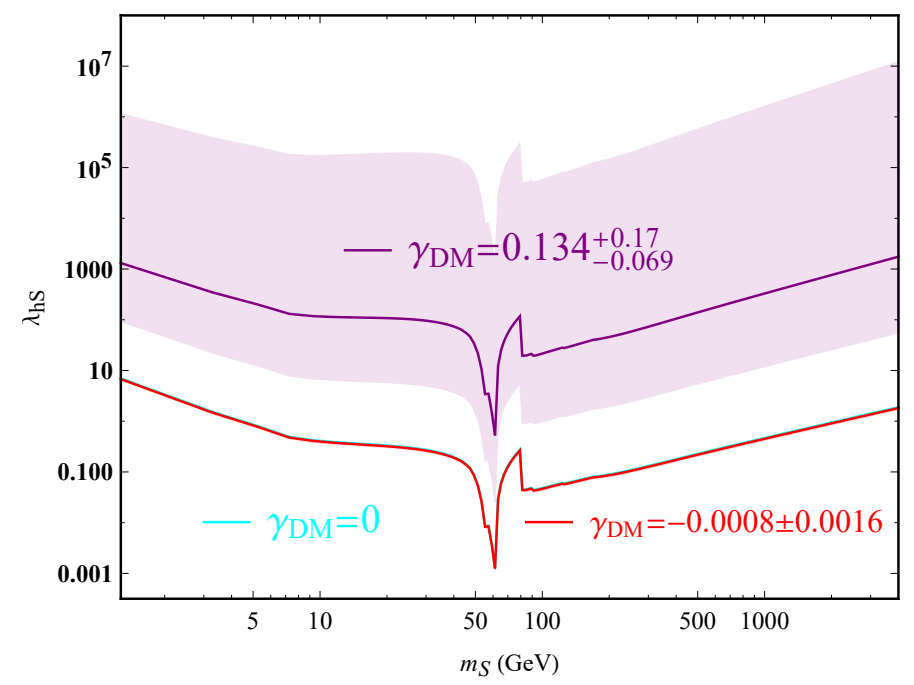

Figure 5. Allowed parameter regions in the $\left(m_{\mathcal{S}}-\lambda_{h \mathcal{S}}\right)$ plane under the constraint of dark matter relic density $\Omega h^{2}=0.1198 \pm 0.0012[12]$.

For this scalar DM model, one can straightforward get the scalar DM relic density by inserting eq. (5.3) into eq. (4.6). We find that only the DM mass $m_{\mathcal{S}}$, the coupling strength $\gamma_{\mathrm{DM}}$ and the coupling constant $\lambda_{h \mathcal{S}}$ are sensitive to the scalar DM relic density.

We show the allowed parameter regions in the $\left(m_{\mathcal{S}}-\lambda_{h \mathcal{S}}\right)$ plane in figure 5 , where the bounds come from the DM relic density eq. (5.11). In figure 5, the cyan shad bands represent $\gamma_{\mathrm{DM}}=0$ that means there is a vanishing interaction between $\mathrm{DE}$ and scalar DM. The purple shad bands represent $\gamma_{\mathrm{DM}}=0.134_{-0.069}^{+0.17}$ case which is obtained from the $(\mathrm{SGL}+\mathrm{SNe}+\mathrm{Hz})$ cosmological observations bounds. In other words, there is an interaction between DE and scalar DM, and the energy of DE converts into that of the scalar DM. And $\lambda_{h \mathcal{S}}$ decreases compared to the $\gamma_{\mathrm{DM}}=0$ case when scalar DM relic density limit is taken into account. All the solid lines here represent the central values.

The red shad bands represent the case of $\gamma_{\mathrm{DM}}=-0.0008 \pm 0.0016$ that is obtained by the limitation of the cosmological observation amount $(\mathrm{CMB}+\mathrm{BAO}+\mathrm{SNe})$. The negative coupling $\gamma_{\mathrm{DM}}$ means not only there is an interaction between DE and DM but also the energy of DM flows to that of the DE. After taking into account the limitation of the relic density of DM, $\lambda_{h \mathcal{S}}$ will be increased compared with the vanishing interaction scenario. The coupling of Higgs and scalar DM $\lambda_{h \mathcal{S}}$ is too large to be reasonable and even is excluded completely, which can also be seen from figure 5 .

It should be emphasized that although the $\gamma_{\mathrm{DM}}$ obtained by the cosmological observation of $(\mathrm{SGL}+\mathrm{SNe}+\mathrm{Hz})$ is large and beyond the range of $x_{f}$ set in the previous section, we found that in this case, $x_{f}$ still remains around 20.

In addition, we can see that when all the lines have an obvious jump at $m_{s} \approx 80 \mathrm{GeV}$, the reason is that the $\mathcal{S S} \rightarrow W^{+} W^{-}$channel annihilation will be opened when $s>2 m_{W}$. The contribution of this annihilation channel is larger and the discontinuity is very obvious. In fact, there will be a break in the lines as long as a channel is closed, which is not obvious due to the little difference in contribution between adjacent channels. 


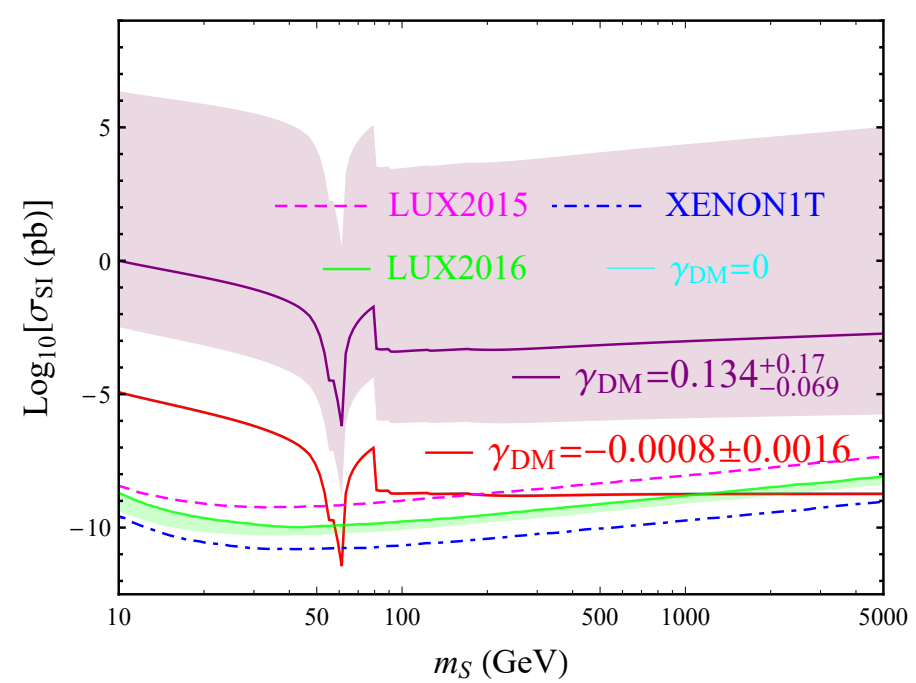

Figure 6. DM-nucleon scattering cross section $\sigma_{S I}$ as the function of DM mass for several $\gamma_{\mathrm{DM}}$. The magenta dashed line, purple line, and blue dot-dashed line represent the bounds from the LUX2015 [108], LUX2016 [108] and Xenon1T [108], respectively. And the purple shad band refers to $2 \sigma$ deviation to their central values that were chosen by authors in refs. [104, 109].

\subsection{DM direct detection}

The spin-independent DM-nucleon scattering cross section for the scalar DM model has the form of [104]:

$$
\sigma_{\mathrm{SI}}=\frac{\lambda_{h \mathcal{S}}^{2} f_{N}^{2} \mu^{2} m_{N}^{2}}{4 \pi m_{h}^{4} m_{\mathcal{S}}^{2}}
$$

where the DM-nucleon reduced mass $\mu=m_{N} m_{\mathcal{S}} /\left(m_{N}+m_{\mathcal{S}}\right)$ with $m_{N}$ being the nucleon mass, the Higgs mass $m_{h}=125 \mathrm{GeV}[105,106]$, the hadron matrix element $f_{N} \simeq 0.3$ [107]. $m_{\mathcal{S}}$ and $\lambda_{h \mathcal{S}}$ are the DM mass and the coupling constant respectively, and they depend on the constructed scalar DM model.

The spin-independent DM-nucleon mesh cross section as a function with $m_{\mathcal{S}}$ is shown in figure 6. The limitations of the direct DM detection experiments include LUX2015, LUX2016, XENON1T, which are marked by the magenta dashed, green shaded bands, and blue dot-dashed line, respectively. While the cyan, red, and purple shaded bands are the $\gamma_{\mathrm{DM}}=0, \gamma_{\mathrm{DM}}=-0.0008 \pm 0.0016$ and $\gamma_{\mathrm{DM}}=0.134_{-0.069}^{+0.17}$ cases, respectively. Note that the cyan shaded bands are completely covered by red shaded bands.

Compared with $\gamma_{\mathrm{DM}}=0$, i.e., the $\mathrm{DM}$ and $\mathrm{DE}$ have vanishing interaction, figure 6 indicates that:

- When $\gamma_{\mathrm{DM}}=0.134_{-0.069}^{+0.17}$, the feasible parameter spaces of spin-independent DMnucleon cross section shifts upward, after considering both the direct detection experiments and the DM relic density bounds, the scalar DM model will be completely eliminated. 


\begin{tabular}{|ccc|}
\hline Viable regions & Resonant mass regions & High mass regions \\
\hline$\gamma_{\mathrm{DM}}=0.134_{-0.069}^{+0.17}$ & - & - \\
$\gamma_{\mathrm{DM}}=0$ & $59 \mathrm{GeV} \lesssim \mathrm{m}_{\mathcal{S}} \lesssim 63 \mathrm{GeV}$ & $m_{\mathcal{S}} \gtrsim 1.5 \mathrm{TeV}$ \\
$\gamma_{\mathrm{DM}}=-0.0008 \pm 0.0016$ & $58.5 \mathrm{GeV} \lesssim \mathrm{m}_{\mathcal{S}} \lesssim 63.5 \mathrm{GeV}$ & $m_{\mathcal{S}} \gtrsim 1.45 \mathrm{TeV}$ \\
\hline
\end{tabular}

Table 3. The viable regions in the resonant mass and high mass regions under the limits of LUX2016 for the three $\gamma_{\text {DM }}$ cases [108].

- When $\gamma_{\mathrm{DM}}=-0.0008 \pm 0.0016$, the shape will be extended. Also, after taking into account the direct detection experiment and DM relic density limitation, the feasible parameter space of the scalar DM model is increased.

For example, under the limits of LUX2016 experiment [108], the increase is not obvious in the resonant mass region, but it is relatively obvious in the large mass regions. More specifically, it can be obtained from table. 3 .

Therefore, if $(\mathrm{SGL}+\mathrm{SNe}+\mathrm{Hz})$ and $(\mathrm{CMB}+\mathrm{BAO}+\mathrm{SNe})$ cosmological observation data are completely credible within the errors, but they lead to a completely contradictory conclusion, then there may be something wrong with the scalar DM model. On the contrary, if the scalar DM dark matter model is correct, then there must be something wrong for the $(\mathrm{SGL}+\mathrm{SNe}+\mathrm{Hz})$ experimental data, while the correctness of the $(\mathrm{CMB}+\mathrm{BAO}+\mathrm{SNe})$ cosmological observation data need to be further confirmed.

\subsection{DM indirect detection}

After discussing the influence of the added interaction term $Q_{\mathrm{DM}}$ on the DM direct detection, in this section, we will turn to its impact on another kind of DM detection, i.e., DM indirect detection. Higgs invisible decay is an important part of the DM indirect detection. According to the potential eq. 5.2, the Higgs invisible decay channel is $h \rightarrow \mathcal{S S}$, and then the corresponding decay width is expressed as:

$$
\Gamma(h \rightarrow \mathcal{S S})=\frac{\lambda_{h \mathcal{S}}^{2} v^{2}}{32 \pi m_{h}} \sqrt{1-\frac{4 m_{\mathcal{S}}^{2}}{m_{h}^{2}}}
$$

To make sense of this equation, in other words, to make Higgs invisible decay $(h \rightarrow \mathcal{S S})$ occur, thus $m_{\mathcal{S}}<1 / 2 m_{h}$.

The interaction term $Q_{\mathrm{DM}}$ will make an effect on the interaction coupling $\lambda_{h \mathcal{S}}$, as can be seen in figure 5. This effect can be further transmitted to the Higgs invisible decay width $(\Gamma(h \rightarrow \mathcal{S S}))$ through the coupling $\lambda_{h \mathcal{S}}$. Applying the total Higgs decay width $\left(\Gamma_{h} \simeq\right.$ $4.15 \mathrm{MeV}$ ), we plot the branching ratio of the Higgs invisible decay in figure 7. In which, we also show the limits from the LHC $B r_{i n v}<16 \%$ [110], ILC $B r_{i n v}<1 \%$ [22], FCC-ee $B r_{i n v}<0.5 \%[23,24]$, and CEPC $B r_{i n v}<0.14 \%[25]$. The case $\left(\gamma_{\mathrm{DM}}=0.134_{-0.069}^{+0.17}\right)$ is almost excluded by all the experiments, while for the remaining two cases, the experimental limits ILC, FCC-ee, and CEPC lead to $58.5 \mathrm{GeV} \lesssim \mathrm{m}_{\mathcal{S}} \lesssim 62.5 \mathrm{GeV}$. Those are almost consistent with DM direct experiment (see table 3 ). 


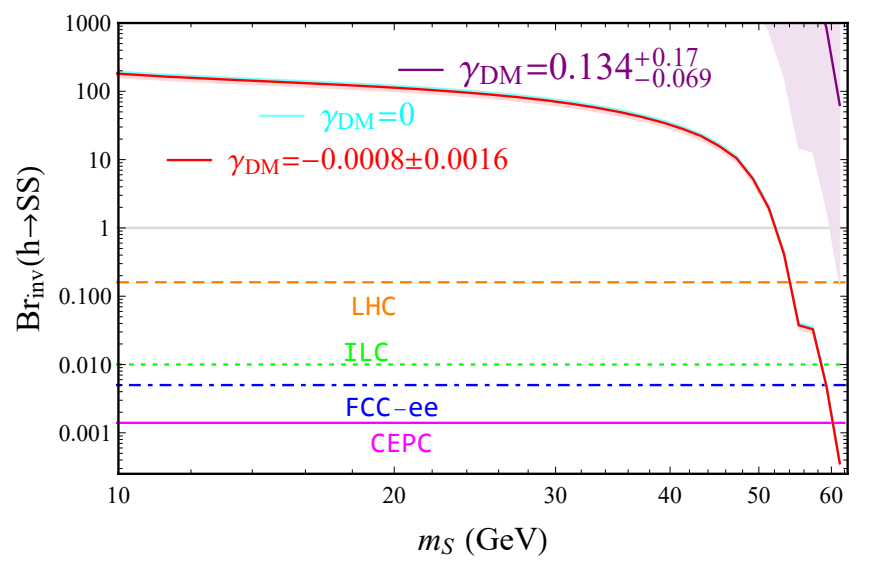

Figure 7. The branching ratio of the Higgs invisible decay.

\section{Summary}

In this paper, motivated by the simplest interaction model between DE and DM with interaction term $Q=3 \gamma_{\mathrm{DM}} H \rho_{\mathrm{DM}}$, we construct the corresponding interaction model of DE and WIMP DM, in which the WIMP DM annihilation term and the interaction term between DM and DE are considered simultaneously. We then discuss the freeze-out parameter $x_{f}=m_{\mathrm{DM}} / T$ with $T$ being the DM freeze-out temperature and deduced the WIMP DM relic density in this new scenario. The resultant DM relic density will be magnified by $\frac{2-3 \gamma_{\mathrm{DM}}}{2}\left[2 \pi g_{*} m_{\mathrm{DM}}^{3} /\left(45 s_{0} x_{f}^{3}\right)\right]^{\gamma_{\mathrm{DM}}}$ times. The new DM relic density opens a new way to test WIMP DM models through the interaction strength $\gamma_{\mathrm{DM}}$, using the observational constraints from the latest cosmological data.

For the interaction strength $\gamma_{\mathrm{DM}}$, we employ cosmological observations to constrain the interaction strength $\gamma_{\mathrm{DM}}$ and find that different observations may make a significant difference for the interaction strength $\gamma_{\mathrm{DM}}$. Specifically, $(\mathrm{SGL}+\mathrm{SNe}+\mathrm{Hz})$ and $(\mathrm{CMB}+\mathrm{BAO}+\mathrm{SNe})$ cosmological observation data will give out $\gamma_{\mathrm{DM}}=0.134_{-0.069}^{+0.17}$ and $\gamma_{\mathrm{DM}}=-0.0008 \pm 0.0016$ respectively. When the interaction strength is $\gamma_{\mathrm{DM}}=0$, the interaction will vanish, and the model will reduce to the standard $\Lambda$ CDM model.

As an example, we analyze the case where WIMP DM is a scalar DM. After further considering the constraints from the DM direct detection experiment, DM indirect detection experiments and DM relic density, we observe that the allowed parameter space of the scalar DM model will be completely excluded for $(\mathrm{SGL}+\mathrm{SNe}+\mathrm{Hz})$ cosmological observation data, while it will be increased for $(\mathrm{CMB}+\mathrm{BAO}+\mathrm{SNe})$ cosmological observation data. Those two cosmological observation data lead to a paradoxical conclusion. Thus, more accurate predictions for $\gamma_{\mathrm{DM}}$ based on the cosmological observations data will provide us with a possible way to screen the WIMP DM models.

\section{Acknowledgments}

We are grateful to Prof. Yu-Feng Zhou, Shuo Cao and Xiaolei Li for helpful communications and discussions. This work was supported in part by Graduate Research and 
Innovation Foundation of Chongqing, China (Grant No. CYS20272, CYS21327), the China Postdoctoral Science Foundation under Grant No. (2019TQ0329, 2020M670476), and the National Natural Science Foundation of China under Grant No.11947302. JiaWei Zhang was supported by the Natural Science Foundation of Chongqing under Grants No.(cstc2018jcyjAX0713), the Science and Technology Research Program of Chongqing Municipal Education Commission under Grant No. KJQN202001541, and the Research Foundation of Chongqing University of Science and Technology under Grant No. CK2016Z03.

Open Access. This article is distributed under the terms of the Creative Commons Attribution License (CC-BY 4.0), which permits any use, distribution and reproduction in any medium, provided the original author(s) and source are credited.

\section{References}

[1] G.R. Blumenthal, S.M. Faber, J.R. Primack and M.J. Rees, Formation of galaxies and large scale structure with cold dark matter, Nature 311 (1984) 517 [INSPIRE].

[2] M. Davis, G. Efstathiou, C.S. Frenk and S.D.M. White, The evolution of large scale structure in a universe dominated by cold dark matter, Astrophys. J. 292 (1985) 371 [INSPIRE].

[3] D. Clowe et al., A direct empirical proof of the existence of dark matter, Astrophys. J. Lett. 648 (2006) L109 [astro-ph/0608407] [INSPIRE].

[4] Supernova Search Team collaboration, Observational evidence from supernovae for an accelerating universe and a cosmological constant, Astron. J. 116 (1998) 1009 [astro-ph/9805201] [INSPIRE].

[5] Supernova Cosmology Project collaboration, Measurements of $\Omega$ and $\Lambda$ from 42 high redshift supernovae, Astrophys. J. 517 (1999) 565 [astro-ph/9812133] [INSPIRE].

[6] S. Cao, G. Covone, M. Paolillo and Z.-H. Zhu, A multi-wavelength study of the gravitational lens COSMOS J095930+023427, Res. Astron. Astrophys. 13 (2013) 15.

[7] S. Cao and Z.-H. Zhu, Cosmic equation of state from combined angular diameter distances: Does the tension with luminosity distances exist?, Phys. Rev. D 90 (2014) 083006 [arXiv: 1410.6567] [INSPIRE].

[8] S. Cao, X. Zheng, M. Biesiada, J. Qi, Y. Chen and Z.-H. Zhu, Ultra-compact structure in intermediate-luminosity radio quasars: building a sample of standard cosmological rulers and improving the dark energy constraints up to $z \sim 3$, Astron. Astrophys. 606 (2017) A15 [arXiv: 1708.08635] [INSPIRE].

[9] S. Cao, M. Biesiada, J. Qi, Y. Pan, X. Zheng, T. Xu et al., Cosmological investigation of multi-frequency VLBI observations of ultra-compact structure in $z \sim 3$ radio quasars, Eur. Phys. J. C 78 (2018) 749 [arXiv:1708.08639] [inSPIRE].

[10] WMAP collaboration, Nine-year Wilkinson Microwave Anisotropy Probe (WMAP) observations: final maps and results, Astrophys. J. Suppl. 208 (2013) 20 [arXiv:1212.5225] [INSPIRE].

[11] Planck collaboration, Planck 2015 results. XIII. Cosmological parameters, Astron. Astrophys. 594 (2016) A13 [arXiv: 1502.01589] [INSPIRE]. 
[12] Planck collaboration, Planck 2018 results. VI. Cosmological parameters, Astron. Astrophys. 641 (2020) A6 [Erratum ibid. 652 (2021) C4] [arXiv:1807.06209] [INSPIRE].

[13] C.O. Heinke, A. Bahramian, N. Degenaar and R. Wijnands, The nature of very faint X-ray binaries; hints from light curves, Mon. Not. Roy. Astron. Soc. 447 (2015) 3034 [arXiv: 1412.4097] [INSPIRE].

[14] T. Bringmann, X. Huang, A. Ibarra, S. Vogl and C. Weniger, Fermi LAT search for internal bremsstrahlung signatures from dark matter annihilation, JCAP 07 (2012) 054 [arXiv: 1203.1312] [INSPIRE].

[15] C. Weniger, A tentative gamma-ray line from dark matter annihilation at the Fermi Large Area Telescope, JCAP 08 (2012) 007 [arXiv: 1204.2797] [InSPIRE].

[16] O. Macias, S. Horiuchi, M. Kaplinghat, C. Gordon, R.M. Crocker and D.M. Nataf, Strong evidence that the galactic bulge is shining in gamma rays, JCAP 09 (2019) 042 [arXiv: 1901.03822] [INSPIRE].

[17] FERMI-LAT collaboration, Measurement of separate cosmic-ray electron and positron spectra with the Fermi Large Area Telescope, Phys. Rev. Lett. 108 (2012) 011103 [arXiv:1109.0521] [INSPIRE].

[18] XENON1T collaboration, The XENON1T dark matter search experiment, Springer Proc. Phys. 148 (2013) 93 [arXiv:1206.6288] [InSPIRE].

[19] LUX collaboration, First results from the LUX dark matter experiment at the Sanford Underground Research Facility, Phys. Rev. Lett. 112 (2014) 091303 [arXiv:1310.8214] [INSPIRE].

[20] LUX collaboration, Results from a search for dark matter in the complete LUX exposure, Phys. Rev. Lett. 118 (2017) 021303 [arXiv:1608.07648] [INSPIRE].

[21] PANDAX-II collaboration, Dark matter results from first 98.7 days of data from the PandaX-II experiment, Phys. Rev. Lett. 117 (2016) 121303 [arXiv:1607.07400] [InSPIRE].

[22] K. Fujii et al., Physics case for the International Linear Collider, arXiv:1506.05992 [INSPIRE].

[23] TLeP Design Study Working Group collaboration, First look at the physics case of TLEP, JHEP 01 (2014) 164 [arXiv:1308.6176] [INSPIRE].

[24] D. d'Enterria, Physics at the FCC-ee, arXiv:1602.05043 [INSPIRE].

[25] CEPC-SPPC Study Group, CEPC-SPPC preliminary conceptual design report. 1. Physics and detector, IHEP-CEPC-DR-2015-01 (2015).

[26] W. Zimdahl and D. Pavon, Interacting quintessence, Phys. Lett. B 521 (2001) 133 [astro-ph/0105479] [INSPIRE].

[27] C. Feng, B. Wang, E. Abdalla and R.-K. Su, Observational constraints on the dark energy and dark matter mutual coupling, Phys. Lett. B 665 (2008) 111 [arXiv:0804.0110] [INSPIRE].

[28] B. Wang, J. Zang, C.-Y. Lin, E. Abdalla and S. Micheletti, Interacting dark energy and dark matter: observational constraints from cosmological parameters, Nucl. Phys. B 778 (2007) 69 [astro-ph/0607126] [INSPIRE].

[29] B. Wang, Y.-g. Gong and E. Abdalla, Transition of the dark energy equation of state in an interacting holographic dark energy model, Phys. Lett. B 624 (2005) 141 [hep-th/0506069] [INSPIRE]. 
[30] J. Cui and X. Zhang, Cosmic age problem revisited in the holographic dark energy model, Phys. Lett. B 690 (2010) 233 [arXiv:1005.3587] [INSPIRE].

[31] E. Abdalla and B. Wang, The mass and the coupling of the dark particle, Phys. Lett. B 651 (2007) 89 [arXiv:0705.0268] [INSPIRE].

[32] M. Jamil and M.A. Rashid, Interacting dark energy with inhomogeneous equation of state, Eur. Phys. J. C 56 (2008) 429 [arXiv:0803.3036] [InSPIRE].

[33] O. Bertolami, F. Gil Pedro and M. Le Delliou, Dark energy-dark matter interaction and the violation of the equivalence principle from the Abell Cluster A586, Phys. Lett. B 654 (2007) 165 [astro-ph/0703462] [INSPIRE].

[34] M. Szydlowski, Cosmological model with energy transfer, Phys. Lett. B 632 (2006) 1 [astro-ph/0502034] [INSPIRE].

[35] Y. Chen, Z.-H. Zhu, J.S. Alcaniz and Y. Gong, Using a phenomenological model to test the coincidence problem of dark energy, Astrophys. J. 711 (2010) 439 [arXiv:1001.1489] [INSPIRE].

[36] S. Cao, N. Liang and Z.-H. Zhu, Testing the phenomenological interacting dark energy with observational H(z) data, Mon. Not. Roy. Astron. Soc. 416 (2011) 1099 [arXiv:1012.4879] [INSPIRE].

[37] H. Zhang, H. Yu, Z.-H. Zhu and Y. Gong, A quantitative criteria for the coincidence problem, Phys. Lett. B 678 (2009) 331 [arXiv:0906.1357] [INSPIRE].

[38] Y. Zhang and H. Li, A new type of dark energy model, JCAP 06 (2010) 003 [arXiv: 1003.2788] [INSPIRE].

[39] S. Kumar and R.C. Nunes, Observational constraints on dark matter-dark energy scattering cross section, Eur. Phys. J. C 77 (2017) 734 [arXiv:1709.02384] [InSPIRE].

[40] W. Yang, S. Pan, E. Di Valentino, R.C. Nunes, S. Vagnozzi and D.F. Mota, Tale of stable interacting dark energy, observational signatures, and the $H_{0}$ tension, JCAP 09 (2018) 019 [arXiv: 1805.08252] [INSPIRE].

[41] E. Di Valentino, A. Melchiorri, O. Mena and S. Vagnozzi, Interacting dark energy in the early 2020s: A promising solution to the $H_{0}$ and cosmic shear tensions, Phys. Dark Univ. 30 (2020) 100666 [arXiv: 1908.04281] [INSPIRE].

[42] E. Di Valentino, A. Melchiorri, O. Mena and S. Vagnozzi, Nonminimal dark sector physics and cosmological tensions, Phys. Rev. D 101 (2020) 063502 [arXiv:1910.09853] [INSPIRE].

[43] S. Cao and N. Liang, Interaction between dark energy and dark matter: observational constraints from OHD, BAO, CMB and SNe Ia, Int. J. Mod. Phys. 22 (2013) 1350082.

[44] Y. Pan, S. Cao, Y. Gong, K. Liao and Z.-H. Zhu, Testing the interaction model with cosmological data and gamma-ray bursts, Phys. Lett. B 718 (2013) 699 [arXiv:1211.0184] [INSPIRE].

[45] Y. Pan, S. Cao, Y. Gong, K. Liao and Z.-H. Zhu, Testing the interaction model with cosmological data and gamma-ray bursts, Phys. Lett. B 718 (2013) 699 [arXiv:1211.0184] [INSPIRE].

[46] S. Cao, Y. Chen, J. Zhang and Y. Ma, Testing the interaction between baryons and dark energy with recent cosmological observations, Int. J. Theor. Phys. 54 (2015) 1492 [InSPIRE].

[47] J.-J. Cao, Z.-X. Heng, J.M. Yang, Y.-M. Zhang and J.-Y. Zhu, A SM-like Higgs near 125 GeV in low energy SUSY: a comparative study for MSSM and NMSSM, JHEP 03 (2012) 086 [arXiv: 1202.5821] [INSPIRE]. 
[48] Q.-H. Cao, E. Ma and G. Rajasekaran, Observing the dark scalar doublet and its impact on the standard-model Higgs boson at colliders, Phys. Rev. D 76 (2007) 095011 [arXiv:0708.2939] [INSPIRE].

[49] Y. Gao, T. Ghosh, K. Sinha and J.-H. Yu, $\mathrm{SU}(2) \times \mathrm{SU}(2) \times \mathrm{U}(1)$ interpretations of the diboson and Wh excesses, Phys. Rev. D 92 (2015) 055030 [arXiv:1506.07511] [InSPIRE].

[50] H. Terazawa and M. Yasue, Excited gauge and Higgs bosons in the unified composite model, Nonlin. Phenom. Complex Syst. 19 (2016) 1 [arXiv: 1508.00172] [INSPIRE].

[51] X. Liu and Z. Liu, TeV dark matter and the DAMPE electron excess, Phys. Rev. D 98 (2018) 035025 [arXiv:1711.11579] [INSPIRE].

[52] J. Yepes, Top partners tackling vector dark matter, Phys. Lett. B 811 (2020) 135890 [arXiv: 1811.06059] [INSPIRE].

[53] S. Cao, J. Qi, M. Biesiada, J. Li and Z.-H. Zhu, Measuring the viscosity of dark matter halos with strongly lensed gravitational waves, Mon. Not. Roy. Astron. Soc. Lett. 502 (2021) L16.

[54] V. Silveira and A. Zee, Scalar phantoms, Phys. Lett. B 161 (1985) 136 [InSPIRE].

[55] J. McDonald, Gauge singlet scalars as cold dark matter, Phys. Rev. D 50 (1994) 3637 [hep-ph/0702143] [INSPIRE].

[56] C.P. Burgess, M. Pospelov and T. ter Veldhuis, The minimal model of nonbaryonic dark matter: a singlet scalar, Nucl. Phys. B 619 (2001) 709 [hep-ph/0011335] [INSPIRE].

[57] V. Barger, P. Langacker, M. McCaskey, M.J. Ramsey-Musolf and G. Shaughnessy, LHC phenomenology of an extended standard model with a real scalar singlet, Phys. Rev. D 77 (2008) 035005 [arXiv:0706.4311] [INSPIRE].

[58] M. Gonderinger, Y. Li, H. Patel and M.J. Ramsey-Musolf, Vacuum stability, perturbativity, and scalar singlet dark matter, JHEP 01 (2010) 053 [arXiv:0910.3167] [INSPIRE].

[59] H. Han, J.M. Yang, Y. Zhang and S. Zheng, Collider signatures of Higgs-portal scalar dark matter, Phys. Lett. B 756 (2016) 109 [arXiv:1601.06232] [INSPIRE].

[60] H. Wu and S. Zheng, Scalar dark matter: real vs. complex, JHEP 03 (2017) 142 [arXiv:1610.06292] [INSPIRE].

[61] C. Boehm, X. Chu, J.-L. Kuo and J. Pradler, Scalar dark matter candidates revisited, Phys. Rev. D 103 (2021) 075005 [arXiv:2010.02954] [INSPIRE].

[62] Y. Pan, S. Cao and L. Li, Constraints on interacting dark energy from time delay lenses, Int. J. Mod. Phys. D 25 (2015) 1650003 [inSPIRE].

[63] S. Cao, M. Biesiada, R. Gavazzi, A. Piórkowska and Z.-H. Zhu, Cosmology with strong-lensing systems, Astrophys. J. 806 (2015) 185 [arXiv: 1509.07649] [INSPIRE].

[64] Y. Chen, R. Li, Y. Shu and X. Cao, Assessing the effect of lens mass model in cosmological application with updated galaxy-scale strong gravitational lensing sample, Mon. Not. Roy. Astron. Soc. 488 (2019) 3745 [arXiv:1809.09845] [INSPIRE].

[65] J.-J. Wei and X.-F. Wu, An improved method to measure the cosmic curvature, Astrophys. J. 838 (2017) 160 [arXiv:1611.00904] [INSPIRE].

[66] D.M. Scolnic et al., The complete light-curve sample of spectroscopically confirmed SNe Ia from Pan-STARRS1 and cosmological constraints from the combined pantheon sample, Astrophys. J. 859 (2018) 101 [arXiv: 1710.00845] [INSPIRE].

[67] L. Bian et al., The gravitational-wave physics II: progress, arXiv:2106.10235 [INSPIRE]. 
[68] A. Piórkowska-Kurpas et al., Inspiraling double compact object detection and lensing rate: forecast for DECIGO and B-DECIGO, Astrophys. J. 908 (2021) 196 [arXiv:2005.08727] [INSPIRE].

[69] Y. Chen, C.-Q. Geng, S. Cao, Y.-M. Huang and Z.-H. Zhu, Constraints on a $\phi C D M$ model from strong gravitational lensing and updated Hubble parameter measurements, JCAP 02 (2015) 010 [arXiv: 1312.1443] [INSPIRE].

[70] J.-Z. Qi, S. Cao, S. Zhang, M. Biesiada, Y. Wu and Z.-H. Zhu, The distance sum rule from strong lensing systems and quasars - Test of cosmic curvature and beyond, Mon. Not. Roy. Astron. Soc. 483 (2019) 1104 [arXiv:1803.01990] [INSPIRE].

[71] T.-H. Liu, J. Zhang, S. Cao, S. Geng, Y. Liu and X. Ji, Implications from simulated strong gravitational lensing systems: constraining cosmological parameters using Gaussian Processes, Astrophys. J. 886 (2019) 94.

[72] T.-H. Liu, S. Cao, J. Zhang, M. Biesiada, Y. Liu and Y. Lian, Testing the cosmic curvature at high redshifts: the combination of LSST strong lensing systems and quasars as new standard candles, Mon. Not. Roy. Astron. Soc. 496 (2020) 708.

[73] J.-Z. Qi, J.-W. Zhao, S. Cao, M. Biesiada and Y. Liu, Measurements of the Hubble constant and cosmic curvature with quasars: ultracompact radio structure and strong gravitational lensing, Mon. Not. Roy. Astron. Soc. 503 (2021) 2179 [arXiv:2011.00713] [INSPIRE].

[74] Y. Ma et al., Testing cosmic opacity from the combination of strongly lensed and unlensed supernova Ia, Astrophys. J. 887 (2019) 163.

[75] S. Cao, G. Covone and Z.-H. Zhu, Testing the dark energy with gravitational lensing statistics, Astrophys. J. 755 (2012) 31.

[76] Y.-B. Ma, S. Cao, J. Zhang, S. Geng, Y. Liu, T. Liu et al., Implications of the lens redshift distribution of strong lensing systems: cosmological parameters and the global properties of early-type galaxies, Eur. Phys. J. C 79 (2019) 121 [arXiv:1901.09737] [InSPIRE].

[77] S. Geng, S. Cao, Y. Liu, T. Liu, M. Biesiada and Y. Lian, The velocity dispersion function of early-type galaxies and its redshift evolution: the newest results from lens redshift test, Mon. Not. Roy. Astron. Soc. 503 (2021) 1319 [arXiv:2102.12140] [INSPIRE].

[78] S. Cao, M. Biesiada, M. Yao and Z.-H. Zhu, Limits on the power-law mass and luminosity density profiles of elliptical galaxies from gravitational lensing systems, Mon. Not. Roy. Astron. Soc. 461 (2016) 2192 [arXiv:1604.05625] [INSPIRE].

[79] S. Cao, X. Li, M. Biesiada, T. Xu, Y. Cai and Z.-H. Zhu, Test of parametrized post-Newtonian gravity with galaxy-scale strong lensing systems, Astrophys. J. 835 (2017) 92 [arXiv: 1701.00357] [InSPIRE].

[80] S. Cao, J. Qi, Z. Cao, M. Biesiada, J. Li, Y. Pan and Z.-H. Zhu, Direct test of the FLRW metric from strongly lensed gravitational wave observations, Sci. Rept. 9 (2019) 11608.

[81] J. Qi, S. Cao, M. Biesiada, X. Ding, Z.-H. Zhu and X. Zheng, Strongly gravitationally lensed type-IA supernovae: Direct test of the Friedman-Lemaître-Robertson-Walker metric, Phys. Rev. D 100 (2019) 023530 [arXiv:1802.05532] [INSPIRE].

[82] S. Cao, J. Qi, M. Biesiada, X. Zheng, T. Xu and Z.-H. Zhu, Testing the speed of light over cosmological distances: the combination of strongly lensed and unlensed Type Ia supernovae, Astrophys. J. 867 (2018) 50 [arXiv:1810.01287] [INSPIRE].

[83] S. Cao, J. Qi, M. Biesiada, T. Liu and Z.-H. Zhu, Precise measurements of the speed of light with high-redshift quasars: ultra-compact radio structure and strong gravitational lensing, Astrophys. J. Lett. 888 (2020) L25 [INSPIRE]. 
[84] T. Liu, S. Cao, M. Biesiada, Y. Liu, Y. Lian and Y. Zhang, Consistency testing for invariance of the speed of light at different redshifts: the newest results from strong lensing and Type Ia supernovae observations, Mon. Not. Roy. Astron. Soc. 506 (2021) 2181.

[85] M.W. Auger et al., The Sloan Lens ACS Survey. X. Stellar, dynamical, and total mass correlations of massive early-type galaxies, Astrophys. J. 724 (2010) 511 [arXiv:1007.2880] [INSPIRE].

[86] M. Cappellari et al., The SAURON project. 4. The mass-to-light ratio, the virial mass estimator and the fundamental plane of elliptical and lenticular galaxies, Mon. Not. Roy. Astron. Soc. 366 (2006) 1126 [astro-ph/0505042] [INSPIRE].

[87] I. Jorgensen, M. Franx and P. Kjaergaard, Planck 2018 results. VI. Cosmological parameters, Mon. Not. Roy. Astron. Soc. 276 (1995) 4.

[88] Y. Wu et al., Exploring the " $L-\sigma$ " relation of HII galaxies and giant extragalactic HII regions acting as standard candles, Astrophys. J. Lett. 888 (2020) 113.

[89] X. Zheng et al., Multiple measurements of quasars acting as standard probes: model independent calibration and exploring the dark energy equation of states, Science China Phys. Mech. Astron. 64 (2021) 259511.

[90] Y. Liu et al., Model-independent constraints on cosmic curvature: implication from updated Hubble diagram of high-redshift standard candles, Astrophys. J. 901 (2020) 129 [arXiv : 2008. 08378] [INSPIRE].

[91] X. Zheng, S. Cao, Y. Liu, M. Biesiada, T. Liu, S. Geng et al., Model-independent constraints on cosmic curvature: implication from the future space gravitational-wave antenna DECIGO, Eur. Phys. J. C 81 (2021) 14 [arXiv: 2012.14607] [InSPIRE].

[92] X. Zheng, X. Ding, M. Biesiada, S. Cao and Z. Zhu, What are $\operatorname{Omh}^{2}\left(z_{1}, z_{2}\right)$ and $\operatorname{Om}\left(z_{1}, z_{2}\right)$ diagnostics telling us in light of $H(z)$ data?, Astrophys. J. 825 (2016) 17 [arXiv: 1604.07910] [INSPIRE].

[93] J.-Z Qi et al., What is parameterized Om(z) diagnostics telling us in light of recent observations?, Res. Astron. Astrophys. 18 (2018) 66.

[94] A. Lewis and S. Bridle, Cosmological parameters from CMB and other data: a Monte Carlo approach, Phys. Rev. D 66 (2002) 103511 [astro-ph/0205436] [INSPIRE].

[95] A.G. Riess, S. Casertano, W. Yuan, L.M. Macri and D. Scolnic, Large Magellanic Cloud Cepheid standards provide a $1 \%$ foundation for the determination of the hubble constant and stronger evidence for physics beyond $\Lambda$ CDM, Astrophys. J. 876 (2019) 85 [arXiv:1903.07603] [INSPIRE].

[96] X. Zheng, M. Biesiada, S. Cao, J. Qi and Z.-H. Zhu, Ultra-compact structure in radio quasars as a cosmological probe: a revised study of the interaction between cosmic dark sectors, JCAP 10 (2017) 030 [arXiv: 1705.06204] [INSPIRE].

[97] N. Aghanim, Y. Akrami, M. Ashdown, J. Aumont and C. Baccigalupi, Planck 2018 results. VI. Cosmological parameters, Astron. Astrophys. 641 (2020) 6.

[98] F. Beutler, C. Blake, M. Colless, D.H. Jones, L. Staveley-Smith, L. Campbell et al., The 6dF Galaxy Survey: Baryon Acoustic Oscillations and the Local Hubble Constant, Mon. Not. Roy. Astron. Soc. 416 (2011) 3017 [arXiv:1106.3366] [InSPIRE].

[99] A.J. Ross, L. Samushia, C. Howlett, W.J. Percival, A. Burden and M. Manera, The clustering of the SDSS DR7 main Galaxy sample - I. A 4 per cent distance measure at $z=0.15$, Mon. Not. Roy. Astron. Soc. 449 (2015) 835 [arXiv:1409.3242] [InSPIRE]. 
[100] BOSS collaboration, The clustering of galaxies in the completed SDSS-III Baryon Oscillation Spectroscopic Survey: cosmological analysis of the DR12 galaxy sample, Mon. Not. Roy. Astron. Soc. 470 (2017) 2617 [arXiv:1607.03155] [InSPIRE].

[101] M. Blomqvist, H.M. Bourboux, N.G. Busca, V.S. Agathe and J. Rich, Baryon acoustic

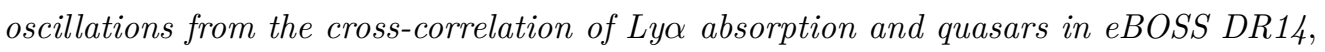
Astron. Astrophys. 629 (2019) 86.

[102] http://hitoshi.berkeley.edu/229C/index.html.

[103] P. Gondolo and G. Gelmini, Cosmic abundances of stable particles: Improved analysis, Nucl. Phys. B 360 (1991) 145 [InSPIRE].

[104] X.-G. He and J. Tandean, New LUX and PandaX-II results illuminating the simplest Higgs-portal dark matter models, JHEP 12 (2016) 074 [arXiv: 1609.03551] [INSPIRE].

[105] ATLAS collaboration, Observation of a new particle in the search for the Standard Model Higgs boson with the ATLAS detector at the LHC, Phys. Lett. B 716 (2012) 1 [arXiv: 1207.7214] [INSPIRE].

[106] CMS collaboration, Observation of a new boson at a mass of $125 \mathrm{GeV}$ with the CMS experiment at the LHC, Phys. Lett. B 716 (2012) 30 [arXiv:1207.7235] [INSPIRE].

[107] J.M. Cline, K. Kainulainen, P. Scott and C. Weniger, Update on scalar singlet dark matter, Phys. Rev. D 88 (2013) 055025 [Erratum ibid. 92 (2015) 039906] [arXiv:1306.4710] [INSPIRE].

[108] XENON collaboration, Physics reach of the XENON1T dark matter experiment, JCAP 04 (2016) 027 [arXiv: 1512.07501] [INSPIRE].

[109] M. Escudero, A. Berlin, D. Hooper and M.-X. Lin, Toward (finally!) ruling out Z and Higgs mediated dark matter models, JCAP 12 (2016) 029 [arXiv:1609.09079] [INSPIRE].

[110] ATLAS and CMS collaborations, Measurements of the Higgs boson production and decay rates and constraints on its couplings from a combined ATLAS and CMS analysis of the LHC pp collision data at $\sqrt{s}=7$ and 8 TeV, JHEP 08 (2016) 045 [arXiv:1606.02266] [INSPIRE]. 